\title{
Thin Anodic Oxide Film Formation and Pitting Corrosion of Nickel in LiCl-KCl Eutectic Containing Oxide Ion
}

\author{
Masayuki TADA and Yasuhiko ITO*
}

\author{
Received January 13, 1992 ; Accepted February 27, 1992
}

\begin{abstract}
In molten $\mathrm{LiCl}-\mathrm{KCl}$ containing oxide ion $\left(\mathrm{O}^{2}\right)$, anodic peak current of nickel was observed in the potential region which was more negative than that of anodic nickel dissolution. This anodic current was associated with oxide ion discharge. XPS analysis showed that this anodic reaction leads to form thin non-stoichiometric oxide film. Thus, this anodic reaction causes passivation, in sense that nickel surface is protected by thin oxide film. This new type passivation behavior of nickel can be termed as "underpotential passivation (UPP)", since it can be observed at more negative potential region than active-passive passivation region. Moreover, pitting corrosion due to thin oxide film breakdown by highly concentrated chloride ion was observed at higher potential region of UPP. Finally, stable region of nickel in molten $\mathrm{LiCl}-$ $\mathrm{KCl}$ eutectic melt was demonstrated on $\mathrm{E}-\mathrm{pO}^{2}$ diagram.
\end{abstract}

\section{INTRODUCTION}

The compatibility of molten salt with constitution materials involves various problems depending on the applications for which the salts are to be used. The most common and important is a corrosion problem.

It is known that corrosion of metals in molten halide systems are often prevented by the formation of oxide film on the metal surface. For example, nickel metal shows high corrosion resistance in molten chloride as well as in molten fluoride, and this is believed to be due to the formation of oxide film on the nickel surface.

However, the mechanism of the formation and the role of this oxide film have not been explained satisfactorily so far. There have been a few works on activepassive passivation of nickel in $\mathrm{LiCl}-\mathrm{KCl}$ eutectic melt ${ }^{1}$ ). On the other hand, several works have been reported on

Department of Nuclear Engineering, Faculty of Engineering, Kyoto University. Yoshidahon-machi, Sakyo-ku, Kyoto 606, Japan.

Key words : nickel, molten chloride, underpotential passivation, pitting corrosion the oxygen evolution reaction from $\mathrm{LiCl}$ $\mathrm{KCl}$ eutectic melt containing oxide ion, at noble metal surfaces ${ }^{2,3)}$. But unfortunately, they did not deal with the behavior of less noble metals, such as $\mathrm{Ni}$, $\mathrm{Fe}, \mathrm{Cr}$ and etc., probably because they are considered to be anodically dissolved into the solution before oxygen evolution occurs ${ }^{4}$. In addition, their treatments do not involve microscopic considerations which are very important to discuss the function of metal surface.

Microscopic considerations are rather involved in the studies conducted in aqueous solution systems. In aqueous solution systems, some excellent microscopic models have been developed ${ }^{5}$. But, in molten chloride systems, the situation is rather different. For example, the temperature is high as $450^{\circ} \mathrm{C}$, and the molten chloride contains almost no water or hydroxide ion.

Taking these backgrounds into account, in this paper, anodic oxide film formation and pitting corrosion of nickel in LiCl-KCl eutectic melt containing oxide ion have been thoroughly investigated. 
It should be emphasized that our main standpoint is to answer the question if oxide film formation occurs at more negative potential than that of anodic dissolution of nickel in molten $\mathrm{LiCl}-\mathrm{KCl}$ eutectic melt containing some amount of oxide ion, even at such a very high chloride concentration as in molten chloride.

\section{EXPERIMENTAL}

The experimental procedures involve in-situ electrochemical measurements and ex-situ surface analyses.

Electrodes were cut in rectangular form $(0.2 \times 8 \times 30 \mathrm{~mm})$ from nickel sheet (Composition is shown in Table.1) and polished with emery paper, then with aluminum powder to obtain mirror-like surface and washed with distilled water, ethanol, and acetone and dried carefully. Reagent grade $\mathrm{LiCl}$ and $\mathrm{KCl}$ (Wako Pure Chemical Industries, Ltd.) were mixed ( $\mathrm{LiCl}: \mathrm{KCl}=59: 41 \mathrm{~mol} \%)$ together and dried in vacuum for several days at $200^{\circ} \mathrm{C}$ to remove water. Salt was melted in a graphite crucible in a stainless steel holder filled with dry argon at $450^{\circ} \mathrm{C}$. Argon gas was bubbled into the melt through an alumina tube for more than one hour to remove residual water further. Pure lithium oxide (99.9\%: Rare Metallic Co., Ltd.) powdered in a drybox with very low water concentration $(<0.5 \mathrm{ppm})$ was used as an oxide ion source. Oxide ion concentration was estimated supposing that all added lithium oxide dissolves and produces oxide ion in molten $\mathrm{LiCl}-\mathrm{KCl}$. Reference

Table.1. Composition of nickel specimen.

\begin{tabular}{|c|c|c|c|}
\hline $\mathrm{Ni}$ & $\mathrm{Mn}$ & $\mathrm{Fe}$ & C \\
\hline$>99.7$ & $<0.03$ & $<0.03$ & $<0.01$ \\
\hline
\end{tabular}

electrode was a $\mathrm{Ag}$ wire immersed in $\mathrm{LiCl}-\mathrm{KCl}$ containing $1 \mathrm{~mol} \% \mathrm{AgCl}$, contained in a pyrex glass tube with thinner bottom. Dynamic reference electrode using electrochemically deposited lithium 6) was used to correct the potential measured by the $\mathrm{Ag} / \mathrm{AgCl}$ reference electrode and all potentials in this paper are referred to this potential. All electrochemical experiments were conducted at $450^{\circ} \mathrm{C}$. Potential sweep method was applied to investigate electrochemical behavior of nickel. Surface analyses were conducted by SEM and XPS.

\section{RESULTS AND DISCUSSIONS}

\subsection{Underpotential passivation (UPP) of nickel.}

Figure 1 shows anodic polarization curve in molten $\mathrm{LiCl}-\mathrm{KCl}$ eutectic melt after lithium oxide addition. Before lithium oxide addition, currents began to increase near $2.3 \mathrm{~V}$, which is due to the occurrence of anodic nickel dissolution. Whereas, after lithium oxide addition, anodic peak indicated as "a" appears at much more negative potential than that of the current increase.

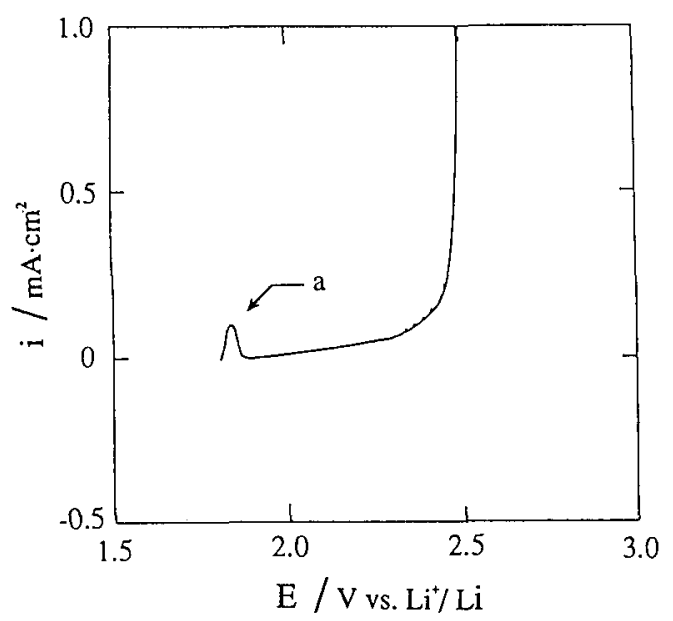

Fig.1 Polarization curve for nickel in molten $\mathrm{LiCl}-\mathrm{KCl}$ eutectic after lithium oxide addition, oxide ion concentration $0.8 \mathrm{~mol} \%$, scan rate $1 \mathrm{mV} \cdot \mathrm{s}^{-1}$ 


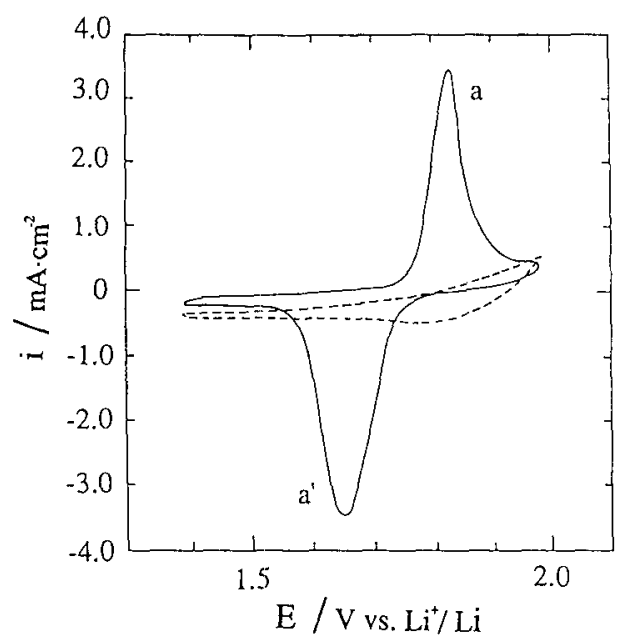

Fig.2 Cyclic voltammograms for nickel in molten $\mathrm{LiCl}-\mathrm{KCl}$ eutectic; (--) before and $(\rightarrow)$ after lithium oxide addition, oxide ion concentration $0.8 \mathrm{~mol} \%$, scan rate $100 \mathrm{mV} \cdot \mathrm{s}$.

To investigate this "peak a" in more detail, cyclic voltammograms were measured as shown in Fig.2. Both peaks a and $\mathrm{a}^{\prime}$ appeared immediately after lithium oxide addition $(0.8 \mathrm{~mol} \%)$, which value increased with time and gradually approached to saturated ones. This indicates that oxide ion affects directly on the electrochemical behavior of nickel in this potential range. Peak a' apparently corresponds to the reverse reaction of peak a. No peaks could be observed in the same potential range on $\mathrm{Pt}$ working electrode. Furthermore, no current perturbation was observed on these peaks with agitation of the solution by argon bubbling. Therefore, peaks a and $a^{\prime}$ apparently reflect the characteristic nature of Ni surface.

Peak currents at $a$ and $a^{\prime}$ increased as oxide ion concentration increased, as shown in Fig.3. Apparently, this behavior differs from the active-passive passivation', because in case of activepassive passivation, peak current should decrease as oxide ion concentration in-

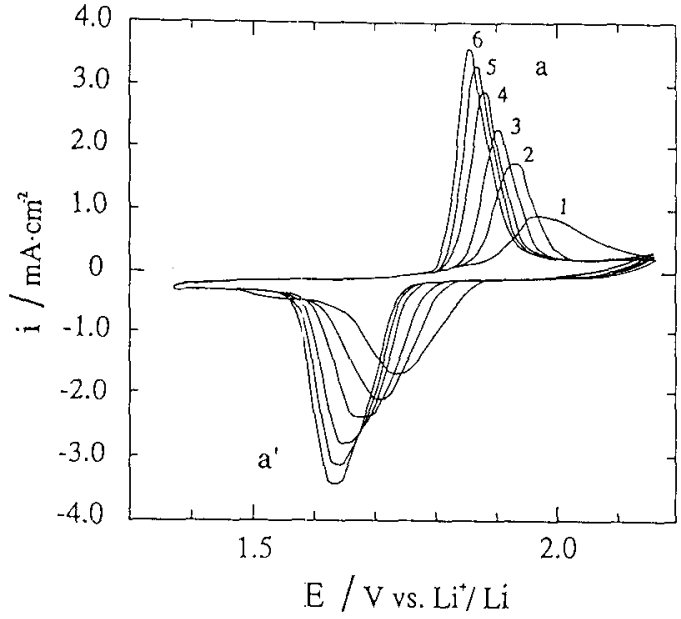

Fig.3 Cyclic voltammograms for nickel in molten $\mathrm{LiCl}-\mathrm{KCl}$ eutectic; (1) $0.05 \mathrm{~mol} \%$, (2) $0.1 \mathrm{~mol} \%$, (3) $0.2 \mathrm{~mol} \%$, (4) $0.4 \mathrm{~mol} \%$, (5) $0.6 \mathrm{~mol} \%$, (6) $0.8 \mathrm{~mol} \%$ oxide ion concentration, scan rate $100 \mathrm{mV} \cdot \mathrm{s}^{-1}$

creases. Potentials at $i=0$ in voltammograms and the rest potentials obtained after releasing the applied potential were in good agreement and these potentials could be considered as quasi-equilibrium potentials corresponding to various oxide ion concentrations. Quasi-equilibrium potentials thus obtained decrease linearly with $\mathrm{pO}^{2}$ as shown in Fig.4. In this figure, oxide ion concentration is expressed with [mol. $\left.\mathrm{kg}^{-1}\right]$ for the convenience of practical use. The slope is $0.071\left(\mathrm{~V} / \mathrm{pO}^{2}\right)$ which is very close to $2.3 \mathrm{RT} / 2=0.072$.

Figure 5 shows voltammograms for nickel obtained after potentiostatic anodic oxidation at $1.85 \mathrm{~V}$. Cathodic peak current $a^{\prime}$ varies with the anodization time before the start of cathodic scan. It should be noticeable that the longer the potentiostatic oxidation time, the more the amount of charge consumed at cathodic reaction of peak $a^{\prime}$, and the more negative potential side the peak potential shifts. This suggests that more charge and energy must be needed for 


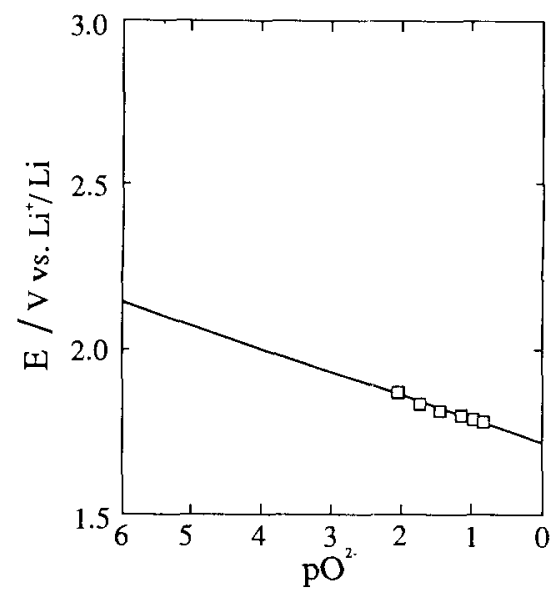

Fig.4 Quasi-equilibrium potentials plotted on E-pO' diagram at $450^{\circ} \mathrm{C}$ ( conc. : $\mathrm{mol} \cdot \mathrm{kg}^{-1}$ ); () experimental results, (-) empirical E-pO ${ }^{2}$ relation; $\mathrm{E}=0.071 \mathrm{pO}^{2}+1.72\left(\mathrm{~V}\right.$ vs. $\left.\mathrm{Li}^{\dagger} / \mathrm{Li}\right)$

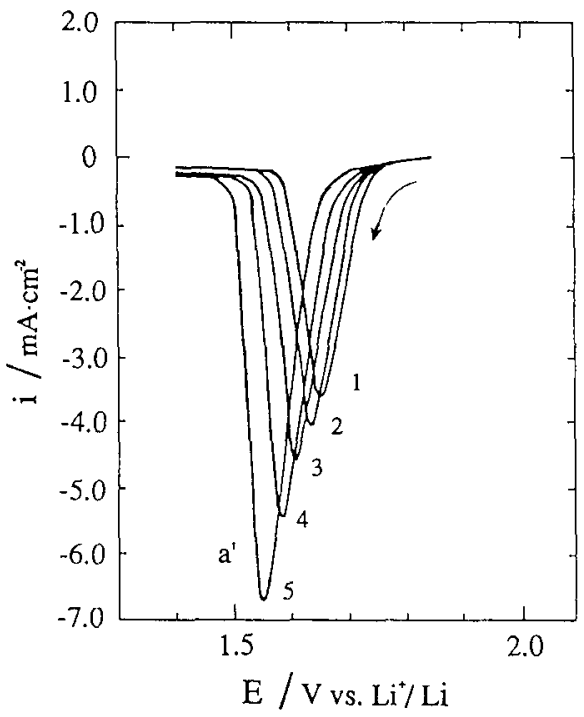

Fig.5 Voltammograms for nickel in molten $\mathrm{LiCl}-\mathrm{KCl}$ eutectic after potentiostatic oxidation at $1.85 \mathrm{~V}$ for (1) $2 \mathrm{~s}$, (2) $5 \mathrm{~s}$, (3) $10 \mathrm{~s}$, (4) $20 \mathrm{~s}$, (5) $60 \mathrm{~s}$, oxide ion concentration $0.8 \mathrm{~mol}$ $\%$, scan rate $100 \mathrm{mV} \cdot \mathrm{s}^{-1}$

reduction since oxidized species is increased and stabilized during the anodic oxidation period.

Nickel surface gradually turned gold during potentiostatic oxidation in the potential region more positive than quasi-equilibrium potential. Whereas, by potentiostatic reduction in the potential region more negative than quasiequilibrium potential, nickel surface kept its original bright surface for long period.

XPS analysis was performed in order to investigate nickel surface change caused by the anodic oxidation. Ar beam sputtering rate in this measurement corresponds to approximately $1-3 \AA \cdot \mathrm{s}^{-1}$ for nickel oxide?). And when original nickel was sputtered for $10 \mathrm{~s}$, O1s signal disappeared and only $\mathrm{Ni2}$ 3/2 signal of
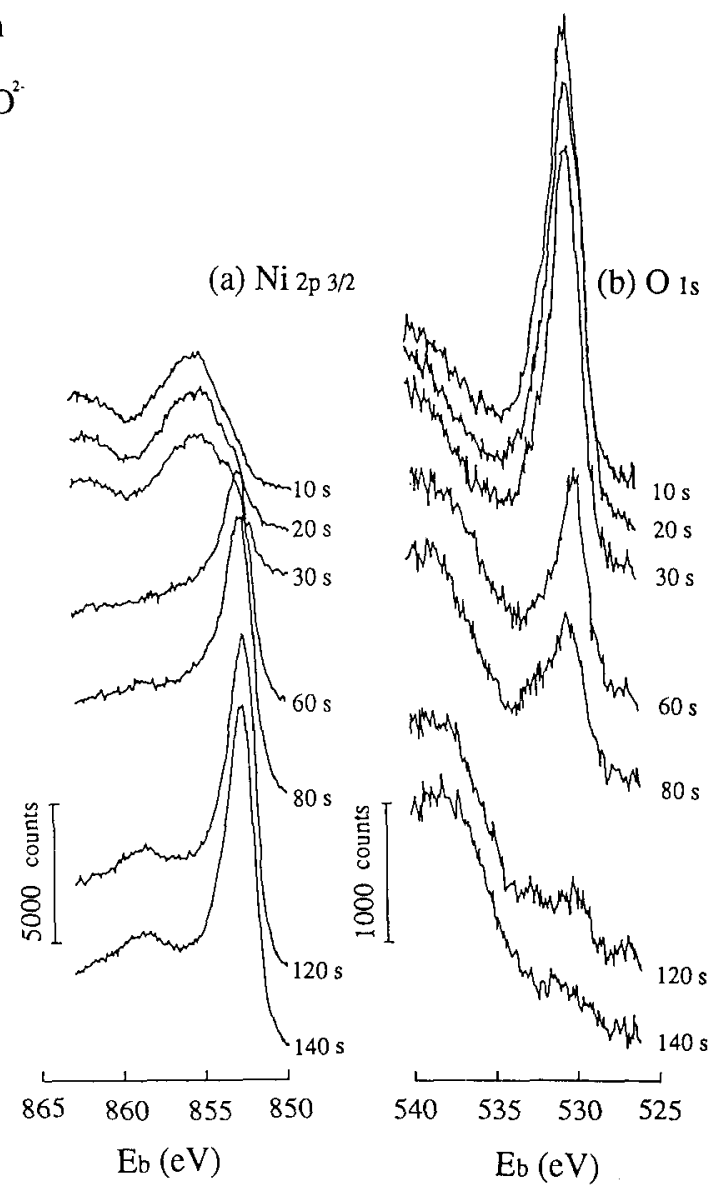

Fig.6 XPS spectra of nickel surface with various argon sputtering time; (a) $\mathrm{Ni} 2 \mathrm{p} 3 / 2$ spectrum and (b) $\mathrm{O}$ is spectrum after $126 \mathrm{~min}$ potentiostatic oxidation at $2.2 \mathrm{~V}$ in $\mathrm{LiCl}-\mathrm{KCl}$ eutectic containing $0.8 \mathrm{~mol} \%$ oxide ion. 
nickel metal could be observed. It means that the signals obtained after $10 \mathrm{~s}$ are all due to the anodic oxidation.

Figure 6 shows XPS spectra of nickel which color turned into gold after potentiostatic oxidation at $2.2 \mathrm{~V}$ for 126 $\min . \mathrm{Li}, \mathrm{K}$, and $\mathrm{Cl}$ were below detection level. It should be noticeable that $\mathrm{O} 1 \mathrm{~s}$ peak is distinctly observed and Ni2p3/2 peak which is indicative of oxidation state is also observed even after $10 \mathrm{~s}$ sputtering. This indicates that nickel oxide film is formed and grown by this anodic oxidation. Also, as sputtering time becomes longer, Ni2p3/2 peak shifts towards Eb value indicating metallic nickel. The peak indicative of nickel metal increases with time to reach saturated value. It is interesting that $\mathrm{O} 1 \mathrm{~s}$ peak can be still observed, even after $\mathrm{Ni} 2 \mathrm{p} 3 / 2$ peak of oxidized state completely shifts towards that of metal. Oxygen to nickel atomic ratio profile estimated by XPS analysis is shown in Fig.7. In the figure, oxidation state of nickel is also given using solid and open circles. The ratio decreases from 1.5 to 0 from nickel oxide surface to nickel substrate with sharp decrease region. This indicates that the oxide film is non-stoichiometric with its composition varying across the film. Also, the thickness of this oxide film can be estimated to be $140-420 \AA$ from this figure. Thus, it is concluded that thin non-stoichiometric nickel oxide film is formed and grown on the surface of nickel specimen by the anodic reaction of peak a. The color change described above can be explained by this conclusion. After this XPS measurement, two color regions were distinctly observed; one of them was metallic nickel surface region sputtered by argon beam, and the other was gold colored surface shielded from argon beam by stainless

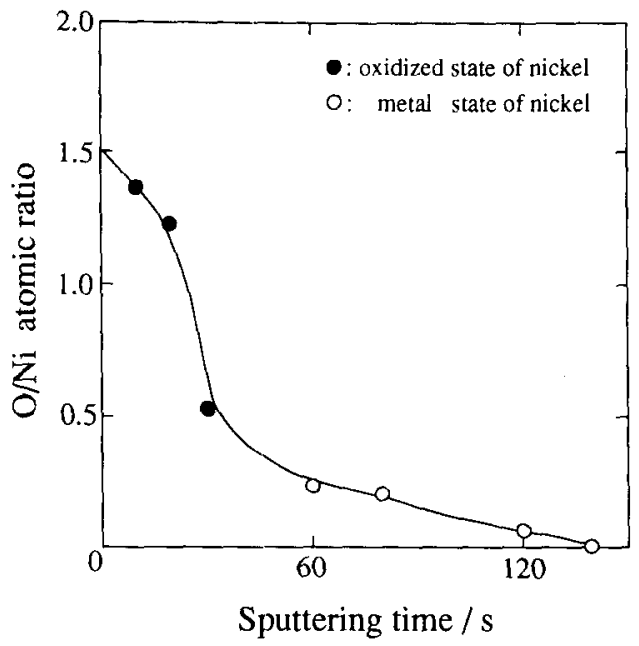

Fig.7 Oxygen to nickel atomic ratio profile measured by XPS with argon ion beam sputtering; after potentiostatic oxidation for 126 min at $2.2 \mathrm{~V}$ in $\mathrm{LiCl}-\mathrm{KCl}$ with $0.8 \mathrm{~mol} \%$ oxide ion concentration.

steel suspender. This means the gold color observed on nickel specimen after potentiostatic oxidation is apparently due to thin oxide film itself.

Since nickel surface is protected by thin oxide film at more negative potential than that of nickel dissolution, this behavior of nickel can be termed as "underpotential passivation (UPP)" to distinguish from well-known activepassive passivation involving nickel dissolution ${ }^{1)}$. This behavior rather resembles protective thin anodic oxide film formations of noble metals prior to oxygen evolution in aqueous solution ${ }^{8}$.

From the experimental results and discussions described above, reaction scheme of UPP can be proposed as in the following: In the first step, chemisorbed oxide ion is discharged to form oxygen atom;

$$
\left.\mathrm{O}^{2-} \text { (on } \mathrm{Ni}\right)=\mathrm{O}(\text { on } \mathrm{Ni})+2 \mathrm{e}^{-}
$$

It should be stressed that this discharge of oxide ion occurs at more negative 
potential than that of anodic dissolution of nickel. The second one is a surface reaction where chemical bonding between oxygen and nickel is formed;

$\mathrm{O}($ on $\mathrm{Ni})+\mathrm{Ni}=\mathrm{Ni}-\mathrm{O}$

Oxygen atoms have the ability to pull electrons from nickel atom. That is why $\mathrm{Ni2p3/2}$ peak of oxidized nickel can be observed near surface region. The third one is a solid reaction involving interdiffusion of oxygen and nickel caused by point defects transportation which motive forces are both concentration gradient and high electric field existing in the film ${ }^{9}$.

The amount of charge corresponding to peak a in Fig.2 was estimated to be about $3 \mathrm{mCcm}-2$. This produces $9 \times 10^{15}$ $\mathrm{cm}^{-2}$ oxygen. Assuming that oxide atoms produce stoichiometric oxide layer, corresponding thickness is estimated to be $11 \AA$. It should be emphasized that oxide film formed by UPP can be considered to be intrinsically more coherent, that is, more protective, compared to the oxide formed by active-passive passivation with dissolution-precipitation mechanism.

\subsection{Pitting corrosion of underpo- tential passivated nickel.}

Figure 8 shows anodic polarization curves of nickel after subjected to potentiostatic passivation for $1 \mathrm{~min}$ and $60 \mathrm{~min}$, respectively, in the UPP region. Currents began to increase with ripples during anodic scan in both curves. After anodic polarization experiments, pitting corrosion was observed on nickel which surface was covered with oxide film (Fig.9). Therefore, current increases with ripples indicate the occurrence of pitting corrosion. Figure 8 also represents that the longer the passivation time is, at the more positive potential pitting corrosion begins. This means that as passivation

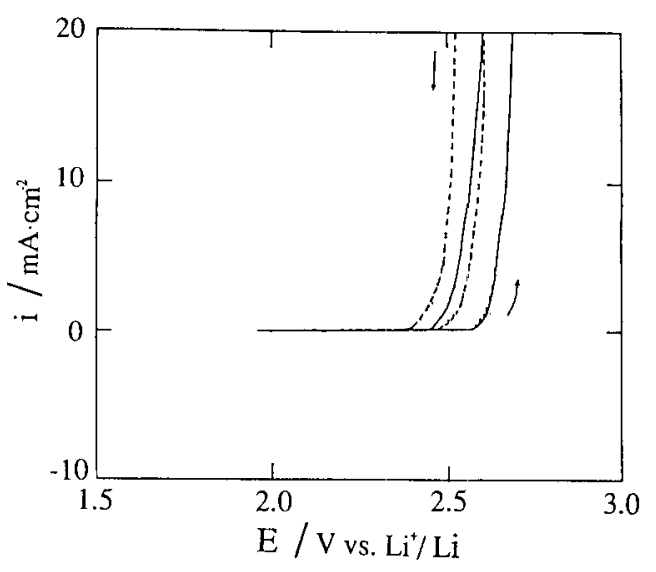

Fig. 8 Polarization curves for nickel in molten $\mathrm{LiCl}-\mathrm{KCl}$ eutectic after UPP at $1.94 \mathrm{~V}$; for $(--) 1 \mathrm{~min}$ and (-) $60 \mathrm{~min}$, oxide ion concentration $0.8 \mathrm{~mol} \%$, scan rate $10 \mathrm{mV} \cdot \mathrm{s}^{-1}$

proceeds, ie., thin oxide film grows, the time necessary for thin oxide film breakdown by chloride ion becomes longer as expected from the case of pitting corrosion in aqueous solution. In case of the cathodic scan, currents due to pitting corrosion decreased rapidly and finally approached zero. This behavior indicates that nickel dissolution ceases to continue at the corresponding potential

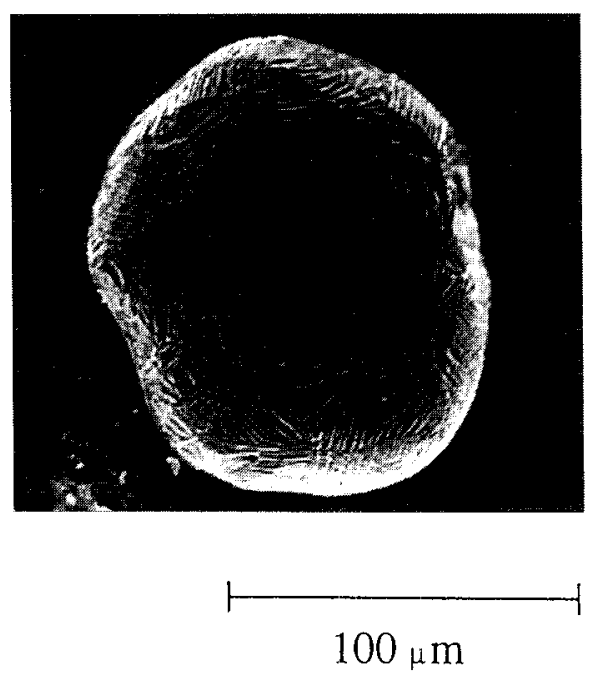

Fig.9 SEM image of typical pitting corrosion observed on nickel after experiment in Fig.8. 


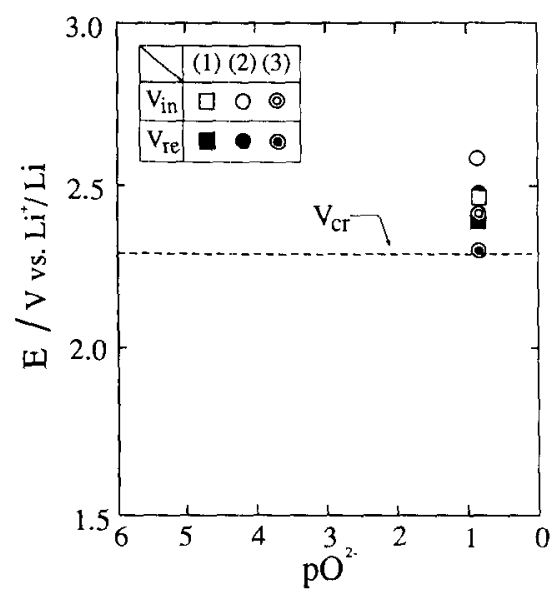

Fig.10 Pitting initiation potentials $\left(V_{\text {in }}\right)$ and repassivation potentials $\left(\mathrm{V}_{\mathrm{re}}\right)$ estimated by anodic polarization curves; after (1) UPP at $1.94 \mathrm{~V}$ for $1 \mathrm{~min},(2) \mathrm{UPP}$ at $1.94 \mathrm{~V}$ for 60 min, (3) UPP in short period (only during anodic scan after reduction at $1.48 \mathrm{~V}$ for 60 $\min ),(--)$ speculated critical potential $\left(V_{c r}\right)$ for pitting corrosion.

(potential at $\mathrm{i}=0$ in Fig.8), where nickel is repassivated.

Both pitting initiation potentials and repassivation potentials at various conditions are plotted in Fig.10. In order to obtain the most negative potential of pitting corrosion and repassivation, the experimental condition denoted by (3) in Fig. 10 was adopted. The condition is the most suitable to remove pre-formed surface oxide sufficiently. Critical potential of pitting corrosion can be speculated as horizontal dashed line in Fig.10. In aqueous solution systems, empirical relationship between critical potential $\left(\mathrm{V}_{\mathrm{cr}}\right)$ and chloride ion activity $\left(a_{C 1}\right)$ is given as;

$\mathrm{V}_{\mathrm{cr}}=\mathrm{A}-\mathrm{B} \log \mathrm{a}_{\mathrm{Cl}}$.

where $\mathrm{A}$ and $\mathrm{B}$ are constants ${ }^{5)}$. Assuming this relation is valid also in the chloride system, $V_{c r}$ can be regarded as almost constant in the oxide ion concentration range shown in the figure, although more systematic experiments should be necessary for the other $\mathrm{pO}^{2-}$ values.

\subsection{Stable condition of nickel in LiCl-KCl.}

From experimental results and discussions on UPP and pitting corrosion described above, E-pO diagram of nickel in $\mathrm{LiCl}-\mathrm{KCl}$ for practical use can be drawn as Fig.11. In the figure, corrosion region is determined by thermodynamic calculation using standard chemical potential of oxide ion ${ }^{10)}$, chemical potential of nickel ion in the melt ${ }^{4}$, and chemical potential of nickel oxide which is calculated by quasi-equilibrium potential supposing stoichiometric oxide is formed electrochemically. Solubility product of thin anodic nickel oxide film is thus estimated to be $10^{-9.2}\left[\mathrm{~mol}^{1} \mathrm{~kg}^{-1}\right]^{2}$. This value is much smaller than that of $10^{-5.3}$

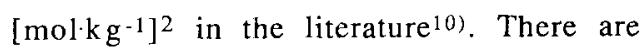
four regions: immunity, UPP, pitting corrosion, and corrosion. Stable region of nickel in $\mathrm{LiCl}-\mathrm{KCl}$ containing oxide ion can be more appropriately estimated by Fig.11 than estimated by conventional thermodynamic calculations in which thermochemical data of stoichiometric nickel oxide is used ${ }^{10}$ ). One can expect stable state of nickel protected by thin

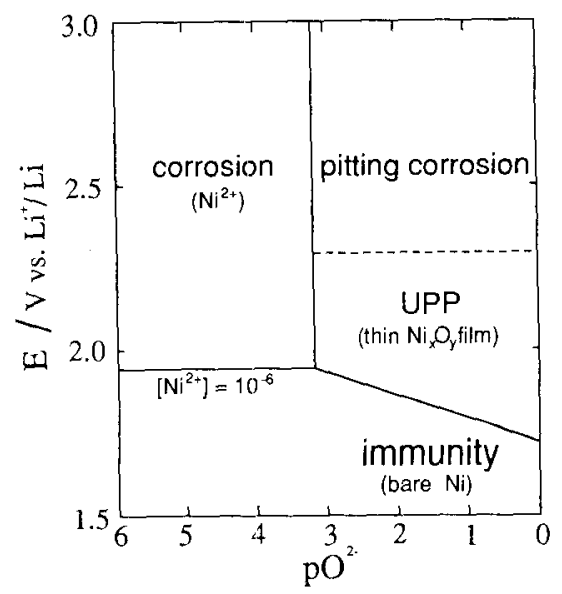

Fig. 11 Practical E -pO diagram for $\mathrm{Ni}-\mathrm{O}$ system in $\mathrm{LiCl}-\mathrm{KCl}$ eutectic at $450^{\circ} \mathrm{C}$ (conc. : $\mathrm{mol} \cdot \mathrm{kg}^{-1}$ ). 
oxide film in the UPP region and severe pitting corrosion region. Also, if one can estimate concentration of impurity oxide ion in $\mathrm{LiCl}-\mathrm{KCl}$ eutectic, surface state of nickel can be speculated by using practical E-pO diagram shown in Fig.11.

\section{CONCLUSION}

In molten $\mathrm{LiCl}-\mathrm{KCl}$ containing oxide ion, anodic current peak associated with oxide ion discharge on nickel could be observed at more negative potential than that of anodic dissolution of nickel. Thin coherent non-stoichiometric oxide formation with its composition varying across oxide film is finally formed by this anodic reaction. Since nickel surface is protected by this thin oxide film at more negative potential than that of anodic nickel dissolution, this new type behavior of nickel can be termed as "underpotential passivation (UPP)". Reaction scheme of UPP has been proposed; discharge of oxide ion to form oxygen atom is the first step. It is stressed that this discharge of oxide ion occurs at more negative potential than that of anodic dissolution of nickel into the melt. The second one is a surface reaction to form chemical bonding between oxygen and nickel. The third one is a solid reaction involving interdiffusion of oxygen and nickel to form thin coherent non-stoichiometric oxide film. In addition, in a upper region of UPP, pitting corrosion was observed. Practically useful $\mathrm{E}-\mathrm{pO}^{2-}$ diagram of nickel in $\mathrm{LiCl}-\mathrm{KCl}$ was proposed. Stable region of nickel in $\mathrm{LiCl}-\mathrm{KCl}$ can be estimated by this practical E-pO ${ }^{2}$ diagram. The method described in this paper would be available in the study of anodic film formation and pitting corrosion of the other metals and alloys in halide systems containing oxide ion.

The present work was financially supported in part by Grant-in-Aid of Science Research from Ministry of Education, Science and Culture, Japan.

\section{REFERENCES}

1) D.L. Piron, S. Asakura and K. Nobe, J. Electrochem. Soc., 123, 503 (1976).

2) D. Inman and M.J. Weaver, J. Electroanal. Chem., 51, 45 (1974).

3) Y. Kanzaki and M. Takahashi, J. Electroanal. Chem., 58, 339 (1975).

4) A.J. Bard, Ed., "Encyclopedia of Electrochemistry of the Elements, vol.10", Marcel Dekker (1976).

5) C.Y. Chao, L.F. Lin and D.D. Macdonald, J. Electrochem. Soc., 128, 1187 (1981), 128, 1194 (1981), 129, 1874 (1982).

6) T. Takenaka, Y. Ito and J. Oishi, Denki Kagaku, 53, 476 (1985).

7) R.L. Tapping, R.D. Davidson, T.E. Jackman and J.A. Davies, Surf. and Interface Anal., 11, 441 (1988).

8) P. Delahay, "Double Layer and Electrode Kinetics", John Wiley \& Sons (1965).

9) A.T. Fromhold, Jr. "Theory of Metal Oxidation, vol.1", North-Holland, Amsterdam (1976).

10) N. Masuko, M. Okada and

T. Hisamatu, Yoyuen (Molten Salts), 6, 570 (1963). 